DOI: https://doi.org/10.46296/ig.v4i8edespdic.0053

\title{
ESTUDIO DE LA APLICACIÓN DEL MANTENIMIENTO PREDICTIVO EN MOTORES DIÉSEL EN LA PROVINCIA DE MANABí
}

\section{STUDY OF THE APPLICATION OF PREDICTIVE MAINTENANCE IN DIESEL ENGINES IN THE PROVINCE OF MANABÍ}

\author{
Zambrano-Castro Jonathan Wilmer ${ }^{1}$; Pérez-Guerrero Julio Nolberto ${ }^{2}$ \\ ${ }^{1}$ Estudiante de la Maestría en Mantenimiento Industrial, Mención en Gestión Eficiente de \\ Mantenimiento, Universidad Técnica de Manabí. Portoviejo, Ecuador. Correo: \\ jonathan.zambrano@utm.edu.ec. ORCID ID: https://orcid.org/0000-0002-1197-624X. \\ 2 Docente de la Maestría en Mantenimiento Industrial - Departamento de Mecánica, \\ Universidad Técnica de Manabí. Portoviejo, Ecuador. Correo: julio.perez@utm.edu.ec. \\ ORCID ID: https://orcid.org/0000-0003-2769-3209.
}

\begin{abstract}
Resumen
El mantenimiento predictivo de motores de combustión interna se encuentra en continuo desarrollo y aplicación a nivel mundial, los motores de combustión interna diésel, por sus ventajas operativas tienen un gran peso para la economía mundial y nacional, por ello es necesario un correcto mantenimiento para garantizar su disponibilidad, sin embargo se desconoce el estado de la aplicación de las técnicas del mantenimiento predictivo en motores diésel, por esta razón el objetivo del trabajo es exponer los resultados del estudio exploratorio de la aplicación del instrumento diagnóstico para determinar el estado del mantenimiento predictivo de motores de combustión interna diésel en la provincia de Manabí, particularmente conocer el equipamiento, técnicas y preparación. La investigación se desarrolló de forma integrada por varios métodos como el análisis documental, el sistémico, el análisis y síntesis, la entrevista no estructurada y la observación directa. Para obtener la información de campo se partió del diseñó del instrumento diagnostico con preguntas orientadas a tres variables, se aplicó a la muestra de 24 talleres. Como resultado del estudio se detectó que los centros de servicio automotriz presentan un bajo o nulo nivel de aplicación de técnicas del mantenimiento predictivo, el factor humano de los centros de posventa es profesional mientras los talleres existe un alto índice de personal empírico, que el nivel técnico y tecnológico de la muestra presenta como herramienta más común el escáner y carecen de técnicas como análisis de aceite, gases, vibraciones y termografía, que los conocimientos requeridos para la aplicación de las técnicas se encuentra por debajo de la media regional y a través de la observación directa se logró establecer que las condiciones de infraestructura son aceptables para su incorporación. Finalmente, lo expuesto permite concluir que el estado de la aplicación de las técnicas del mantenimiento predictivo en motores diésel en la provincia de Manabí es poco satisfactorio.
\end{abstract}

Palabras clave: Mantenimiento predictivo, talleres automotrices, tecnificación automotriz, sector automotriz en Manabí, mantenimiento por condición.

\footnotetext{
Abstract

The predictive maintenance of internal combustion engines is in continuous development and application worldwide, diesel internal combustion engines, for their operational advantages have a great weight for the world and national economy, so proper maintenance is necessary to ensure their availability, however the state of the application of predictive maintenance techniques in diesel engines is unknown, for this reason the objective of the work is to present the results of the exploratory study of the application of the diagnostic instrument to determine the state of

Información del manuscrito:

Fecha de recepción: 04 de octubre de 2021.

Fecha de aceptación: 09 de noviembre de 2021.

Fecha de publicación: 08 de diciembre de 2021.
} 
predictive maintenance of diesel internal combustion engines in the province of Manabí, particularly to know the equipment, techniques and preparation. The research was developed in an integrated way by several methods such as documentary analysis, systemic analysis, analysis and synthesis, unstructured interview and direct observation. To obtain the field information, we started from the design of the diagnostic instrument with questions oriented to three variables, it was applied to the sample of 24 workshops. As a result of the study it was detected that the automotive service centers present a low or no level of application of predictive maintenance techniques, the human factor of the after-sales centers is professional while the workshops there is a high index of empirical personnel, that the technical and technological level of the sample presents as the most common tool the scanner and lack techniques as analysis of oil, gases, vibrations and thermography, that the knowledge required for the application of the techniques is below the regional average and through direct observation it was possible to establish that the infrastructure conditions are acceptable for its incorporation. Finally, the above allows us to conclude that the state of the application of predictive maintenance techniques in diesel engines in the province of Manabí is unsatisfactory.

Keywords: Predictive maintenance, automotive workshops, automotive technification, automotive sector in Manabí, maintenance by condition.

\section{Introducción}

Los motores de combustión interna continúan siendo trascendentales para la economía global. (B. T. Martínez, 2005). Actualmente constituyen algo más del $90 \%$ de las ventas del mercado automotor mundial (Estadísticas de ventas | www.oica.net, 2021).Aunque en el mercado de automotores, encabezan las ventas los vehículos propulsados por gasolina, existe demanda de vehículos diésel debido a que son seguros, económicos, potentes, entre otros. (Errasti y otros, 2013) (Rafael \& Hernández, 2015) (Autodoc.es, 2020). Se conoce por (González, 2013) que el 94\% del comercio mundial es impulsado por los motores diésel a través de transporte marítimo y terrestre, entre tanto en la Unión Europea, el 50\% de los autos nuevos funcionan a diésel. En consecuencia, se puede asegurar que el motor diésel es un componente fundamental en el mercado automotor a nivel mundial.

Dada el elevado número de vehículos automotores a diésel que circulan hoy día, es preciso controlar, registrar y analizar periódicamente el comportamiento de los valores de los parámetros principales su funcionamiento para garantizar su disponibilidad. El control de los parámetros de los motores es necesario para evitar desperfectos y pérdidas significativas, de allí que el 
mantenimiento adquiere gran relevancia en especial las que se apoyan en medios tecnológicos sofisticados para el monitoreo de la condición y la detección temprana de posibles averías. (Hidalgo \& Robles, 2016).

Los fundamentos científicos del mantenimiento industrial en general y automotor en particular, así como los métodos y técnicas específicas para su implementación han venido evolucionando década tras década, pasando por diferentes estadios a saber: el tradicional mantenimiento correctivo, el mantenimiento preventivo, hasta llegar al mantenimiento predictivo. El mantenimiento correctivo (Yanes et al., 2019);(B. T. Martínez, 2005);(Garrido, 2011);(Reina-Pérez et al., 2018) concibe que la máquina está en funcionamiento hasta el momento que se produce una avería, el preventivo establece un conjunto de actividades periódicas prefijadas por el fabricante así como ejecutar acciones correctivas si es detectado durante la operación algún desperfecto y finalmente, el mantenimiento predictivo que se fundamenta en la determinación de la condición de una maquina a través de un conjunto de características (operacionales, térmicas, desgaste, vibraciones, pureza, hermeticidad) y sus parámetros en régimen de operación nominal, el patrón observado de las características, cuando la maquina es nueva, dista de los reflejados durante diferentes momento de su operación.(Lucendo, 2019)

Entre las diferentes filosofías de mantenimiento conocidas destaca y se impone progresivamente a nivel mundial el mantenimiento predictivo, dado a que facilita entre otros: Vigilancia de máquinas, protección de máquinas, diagnóstico de fallos, pronóstico de la esperanza de vida de los componentes todo lo cual se traduce en beneficio para la empresa(B. T. Martínez, 2005) ; (Reina- Pérez et al. , 2018) ; («DMantenimientoPredictivo»,2019) ; (Calloni,2004) ; (Fernández, 2005) a saber: a) Reducción de los costos de reparación y mantenimientos, b) incremento de la confiabilidad y la vida de la máquina y plantas, c) aumento de los ingresos promedios, incremento de la seguridad de operación e) disminución de riegos ambientales, etc. 
Actualmente el avance de la tecnología automotriz, permite realizar el monitoreo y diagnóstico efectivo de los diferentes sistemas de una máquina en general y de los motores en particular utilizando diversos equipos y sistemas de diagnóstico disponibles en el mercado. Ello se alcanza empleando de forma integrada y sistémicas varias técnicas y métodos de monitoreo y registro de la condición como indican varios trabajos relacionados con el tema (Bennett, 2017);(Barrios \& Calderón, 2018);(Reina-Pérez et al., 2018);(Implantación De Un Sistema De Mantenimiento Predictivo Basado En Condición En Una Plataforma Naval, s. f.);(Velásquez, 2018); (Ballesteros, 2017); (Cárdenas \& Cevallos, 2017) (Gutiérrez et al., 2017); (Álava, 2020); (Rodríguez, 1998), a saber: análisis de aceite, medición de vibraciones, análisis de gases, termografía y sonometría. Algunas de ellas se comportan como complementarias y aumentan la fiabilidad del diagnóstico.

La caracterización del estado de aplicación de la implementación de las técnicas y procedimientos del mantenimiento predictivo en una región en país es definida (Garrido, 2011) ; (Barrios \& Calderón, 2018) como el establecimiento del conjunto de características y sus parámetros para las condiciones de realización estándares de este proceso de forma tal que permitan identificar sus falencias, fortalezas y proponer acciones de mejora. Conocer el estado de aplicación de las técnicas de mantenimiento en un país, región - localidad, es importante porque permite identificar las capacidades de la región analizada en cuanto tecnología disponible, competencias de los técnicos e ingenieros encargados de la activad, la cultura de los clientes o dueños de vehículos entre otros, pudiendo llegar a conclusiones sobre las variables antes mencionadas y con ello plantear acciones para su fortalecimiento.

Estadísticas de la Asociación de empresas automotrices del Ecuador (AEDE, 2020) registran que en el parque automotor del país existen 333.328 vehículos pesados comerciales (camión, bus y VAN) que funcionan a base combustible diésel, que tienen un papel relevante en la economía del país y los 
sectores involucrados como: transporte de personas, logística y transporte de mercancía, construcción, etc. Por otro lado, estudios como el de (Ortiz, 2015); (Idrovo \& Pugo, 2017), señalan que en el país existe un número considerable de vehículos ligeros, camionetas y furgonetas a diésel que son utilizados para turismo, transporte escolar, empresarial 0 cuenta propia. En este mismo estudio se señala que solo el $20 \%$ de los ecuatorianos realiza el mantenimiento de su vehículo en la concesionaria, bajo la normativa de fábrica establecida para el buen funcionamiento del motor, y que el $80 \%$ restante, lo realiza con el mecánico de su confianza, donde el tipo de actividades de mantenimiento y la frecuencia de se ejecutan a criterio del propietario, sin un enfoque técnico preestablecido.

El análisis de las publicaciones disponibles en la web y otras fuentes de información de los últimos años sobre la actividad del mantenimiento en el Ecuador y de forma particular en la provincia de Manabí revela la inexistencia de este tipo de trabajo, lo cual se desconoce el nivel de aplicación de los métodos y técnicas de mantenimiento predictivo en centros de servicio automotrices, que brindan mantenimiento a vehículos comerciales livianos a diésel, por esa razón el presente trabajo tiene como objetivo determinar el estado de la aplicación de las técnicas del mantenimiento predictivo en motores diésel en la provincia de Manabí.

\section{Materiales y métodos}

La investigación se desarrolló empleando de forma integrada varios métodos e instrumentos entre los que se encuentran el análisis documental, la observación científica, la entrevista y el análisis estadístico de información siguiendo un procedimiento compuesto por cinco etapas de trabajo a saber:

Estudio del estado del arte de las técnicas del mantenimiento predictivo.

> Caracterización del objeto de estudio.

> Desarrollo del instrumento.

> Aplicación del instrumento.

Análisis y discusión de los resultados.

El análisis documental del estado del arte mundial sobre el mantenimiento 
predictivo, conceptos, factores que influyen en su implementación, técnicas empleadas, los motores diésel etc., se llevó a cabo con el propósito de precisar las variables o factores claves a tener presente en la implementación exitosa del mantenimiento predictivo y el diseño de la herramienta para el estudio de campo. La revisión bibliográfica incluyó a más de 50 obras de pertinencia siendo principalmente artículos científicos indexados, libros, citas técnicas de trabajos ejecutados con anterioridad etc.

La caracterización a priori del objeto de estudio efectuado con el propósito de conocer número de automotores en la provincia, marcas y modelos más difundidos, localización y cantidad talleres de mantenimiento entre otras se realizó, a partir del análisis de más de 10 fuentes de información nacionales y locales.

Para el desarrollo de la investigación de campo se partió del diseño de una entrevista basada en la metodología Delphi la cual constó de 15 preguntas cerradas y abiertas. Se formularon cinco preguntas alrededor de los tres factores esenciales para la implementación de las técnicas de mantenimiento predictivo, según se concluyó en el estudio del arte: el humano (años de experiencia, nivel de escolaridad, formación en la actividad, entre otras.), el modelo y técnicas de gestión empleadas (servicio que oferta el taller, frecuencia de solicitudes, averías más frecuentes, procedimientos, método y procedimientos empleados) y los equipos y herramientas disponibles (equipos con los que cuentan, técnicas aplicadas, procedimientos de detección de falla entre otras).

El estudio se desarrolló en la provincia de Manabí. Para la selección de la muestra se tomó como universo el formado por 1019 empresas y propietarios de la provincia de Manabí según los datos estadísticos proporcionados por el INEC; el total de empresas mencionado corresponde a pymes clasificadas entre mediana y pequeña empresa. Los factores determinantes para la selección de la muestra fueron la relevancia en la actividad de mantenimiento y la representatividad a nivel de provincia. En base a ello la muestra se conformó con 24 de centros de servicio automotriz, distribuidos en la 
zona norte y sur de la provincia de los cuales 14 corresponden a centros de posventa de marcas reconocidas del Ecuador y 10 a los establecimientos más importante de los principales cantones.

En cada centro incluido en la muestra se aplicó la entrevista dirigida a profesionales de la ingeniería mecánica y jefes de talleres y además se realizó la observación científica directa para la recopilación de datos de las instalaciones.

Finalmente, los análisis estadísticos de la información de la entrevista se realizaron mediante software estadístico Excel de Office, para luego proceder con la interpretación y el análisis de los resultados obtenidos de la entrevista a los expertos, mismos que se encuentran agrupados en las tres variables acorde a lo mencionado anteriormente.

\section{Resultados y discusión}

Caracterización de la provincia de Manabí.

La provincia de Manabí ocupa un territorio de unos $19427 \mathrm{~km}^{2}$ con una población de 1395249 habitantes, siendo la cuarta provincia del país por extensión, detrás de Pastaza, Morona Santiago y Orellana. («Datos Geográficos», s. f.). La economía en la provincia se desarrolla principalmente en el sector en la categoría de pequeña y mediana empresa, las PYMES en el Ecuador responden a la clasificación bajo la decisión 702, artículo 3 del CAN. Según datos del INEC del 2019 dentro de provincia de Manabí el $35,56 \%$ corresponde al sector económico denominado Comercio, reparación automotores y motocicletas, siendo así, $\quad 27179$ PYMES que se dedican a estas actividades económicas en la provincia; de estas PYMES, solo 1 651 pertenecen al subsector de reparación automotores y motocicletas, lo que corresponde al $6,07 \%$ de la totalidad de PYMES de la provincia, esta subcategoría se subdivide en Mantenimiento y reparación de vehículos automotores, que corresponde a 1 019 establecimientos que representa al $61,7 \%$; de éstas el $2,25 \%$ se encuentran en la categoría de Pequeña empresa siendo un total de 26 establecimientos, mientras que en categoría de Microempresa se 
encuentran 990 establecimientos lo que corresponde a un $97,15 \%$.

Datos de la AEADE correspondientes al 2 020, colocan a Manabí en el quinto lugar en ventas con una participación de vehículos de 3380 unidades lo que corresponden a 3,9 \%, los principales segmentos vendidos se detallan en la figura 1. Cifras del INEC señalan que el parque automotor de la provincia está compuesto por 123545 unidades que fueron matriculadas en el 2020.

Figura 1. Ventas realizadas en el periodo 2020 en la provincia de Manabí.

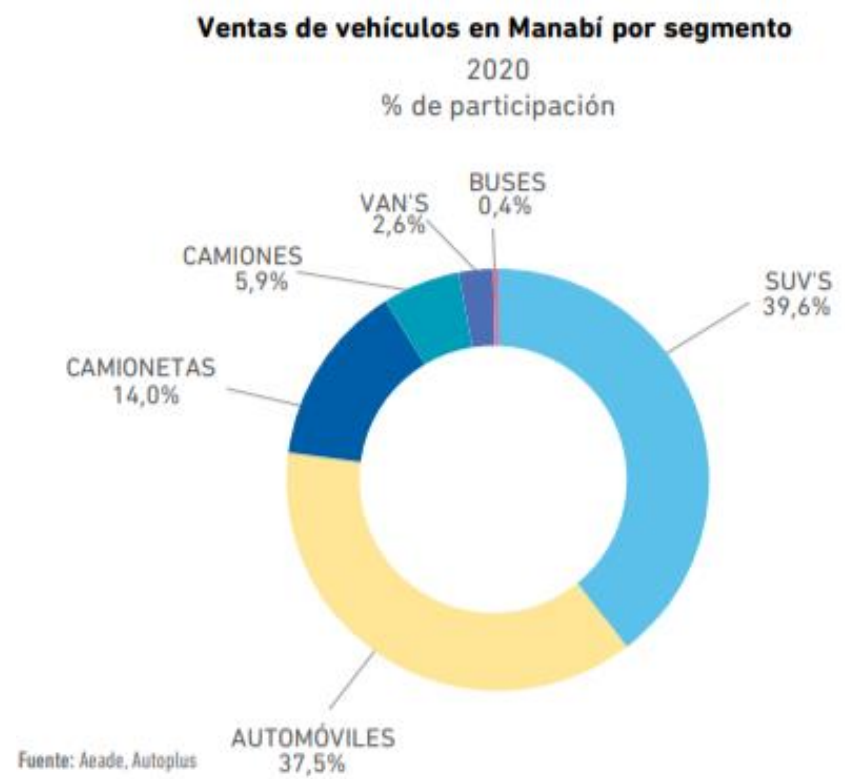

Fuente. (ANUARIO-2020-AEADE-1.pdf, s. f.).

Resultados de la encuesta.

En referencia al factor humano el análisis arrojó que el $100 \%$ del personal de los centros de posventa de automóviles es de sexo masculino, la edad promedio está entre los 30 a 40 años de edad, el $100 \%$ cuenta es graduado técnico o de ingenieros, además todos reciben programas de capacitación avalados de manera recurrente. La práctica en la reparación de motores a diésel oscila entre los 6 a 10 años. Todos los centros investigados poseen al menos un técnico especialista en atención a motores de combustión interna diésel.

En cuanto a los centros de atención pequeños o talleres arrojo que el 100 $\%$ del personal es de sexo 
masculino, se encuentra comprendido entre los 35 a 50 años de edad, el $10 \%$ es graduado de ingeniero, el $30 \%$ posee grado de técnico y el $60 \%$ es de formación empírica. La experiencia en la práctica de diagnóstico y reparación de motores diésel oscila entre 11 a 15 años y es baja su participación en actividades de capacitación.

El análisis de la información de todos las entidades investigadas nos permite afirmar que el personal de servicio de los centros automotrices de Manabí posee una preparación de baja a moderada como lo muestra la figura 2, y de mediana edad, su preparación es baja, con poca participación en programas de capacitación, la figura 3 muestra las áreas de capacitación en las cuales participan con mayor regularidad, en ella se observa que en el área donde se encuentra el mayor interés del personal por capacitaciones, son los Métodos de detección de fallas de motor y sistemas automotrices.

Figura 2. Distribución del personal de los centros de mantenimiento visitados.

d) Personal de formación artesanal (maestro)

c) Obreros o asistente

b) Técnico

a) Ingenieros
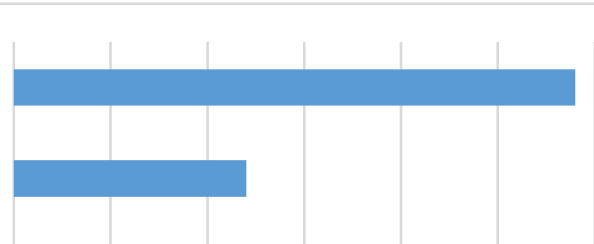

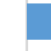

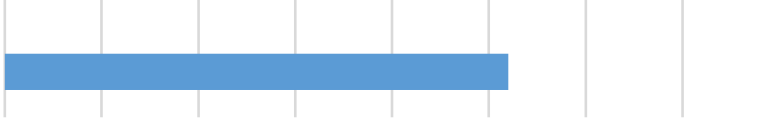

$\begin{array}{lllllllll}0 & 5 & 10 & 15 & 20 & 25 & 30 & 35 & 40\end{array}$

\begin{tabular}{|c|c|c|c|c|c|c|}
\hline & a) Ingenieros & & Técnico & & Obreros o asistente & $\begin{array}{l}\text { d) Personal de } \\
\text { formación artesanal } \\
\text { (maestro) }\end{array}$ \\
\hline Series1 & 26 & & 38 & & 12 & 29 \\
\hline
\end{tabular}

Fuente. Elaboración propia. 
Figura 3. Área de capacitación ofertada al personal de los centros de mantenimiento automotriz.

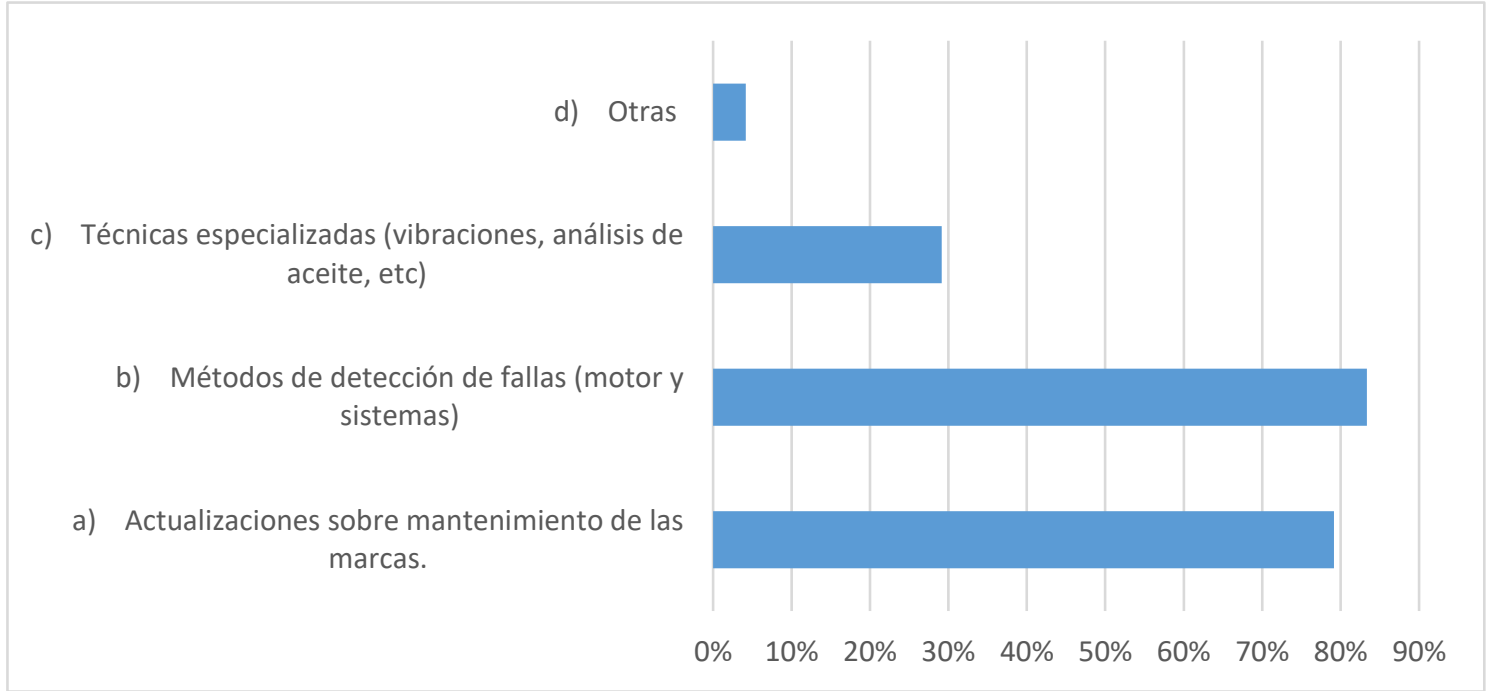

Fuente: Elaboración propia.

En el factor que comprende los modelos y técnicas de gestión de mantenimiento de los talleres, arrojó que el $58 \%$ de las ordenes de trabajo ejecutadas a diario corresponde en primer lugar al $\mathrm{AL}$ mantenimiento preventivo y Correctivo, en segundo lugar, con el $42 \%$ el orden de Mantenimiento Correctivo y Preventivo como se muestra la figura 4.

Figura 4. Solicitudes de trabajo ejecutadas.

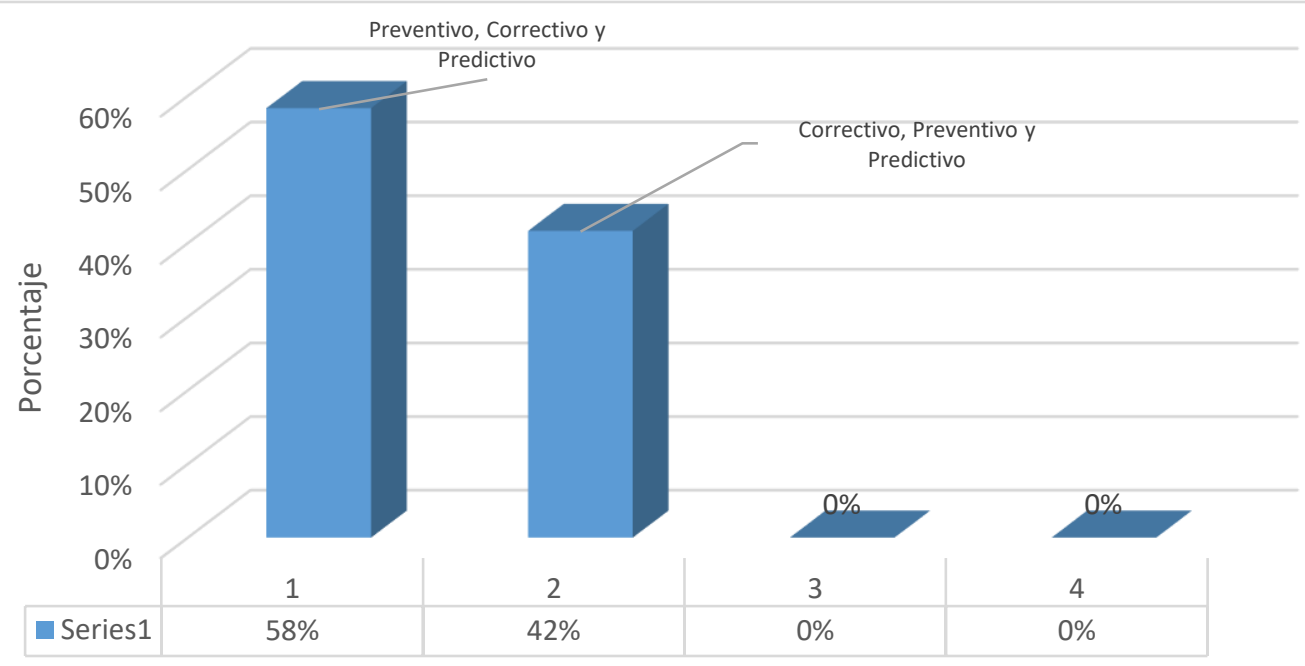

Fuente: Elaboración propia 
La figura 5 muestra los criterios empleados por el personal de servicio investigado para el diagnóstico de una avería o falla, el $96 \%$ utiliza las herramientas como el escáner y multímetro, el $71 \%$ indica que la experiencia y la aplicación de los sentidos como vista, tacto y oído son claves para la determinación de una falla y el $54 \%$ acude a información proporcionada por fabricante 0 sitios web especializados.

Figura 5. Criterios para la determinación de una falla.

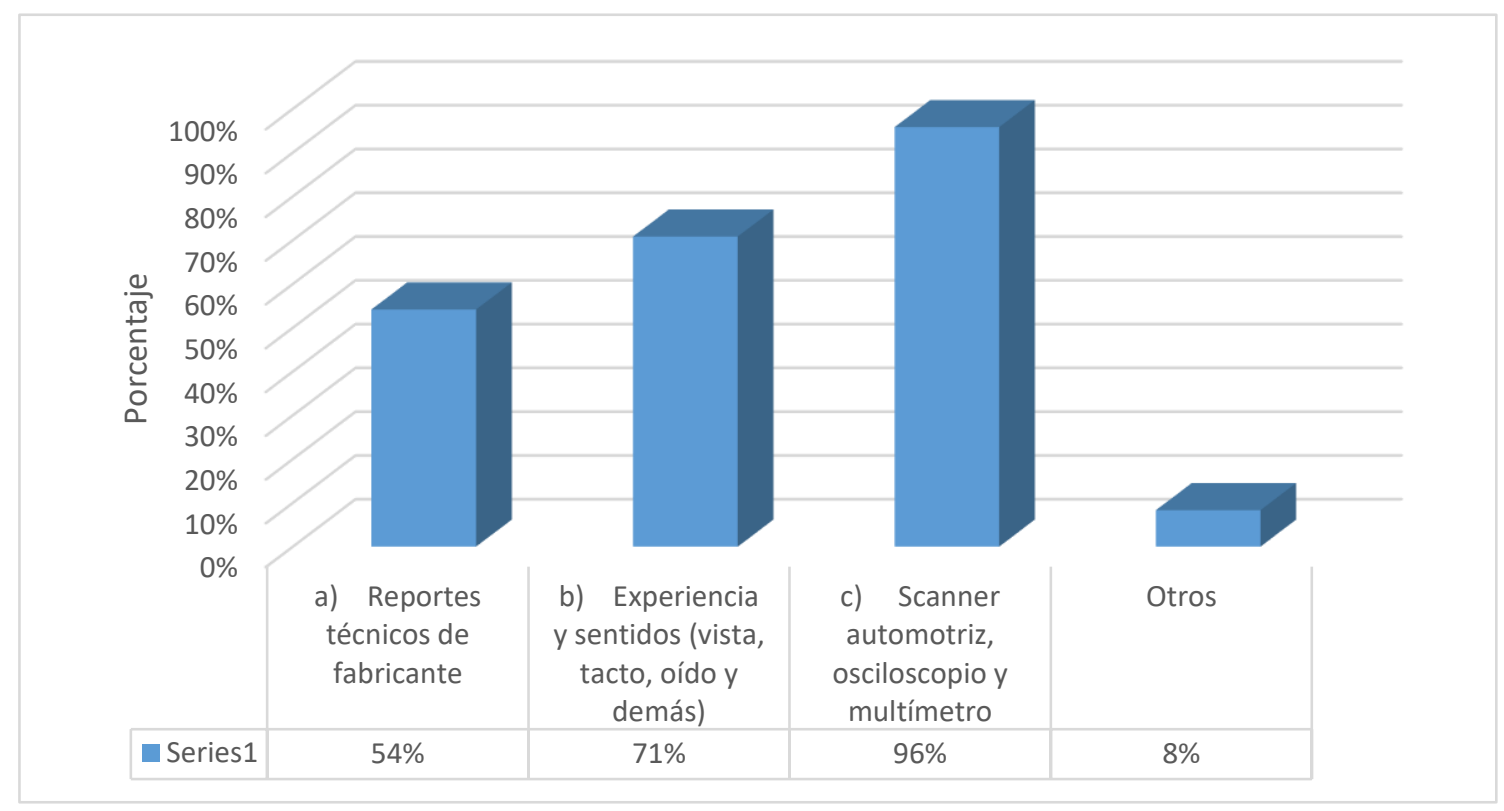

Fuente: Elaboración propia.

En la figura 6 se muestra el gráfico del estado de la aplicación de las técnicas del mantenimiento predictivo con las que cuentan los centros de servicio investigados para el establecimiento de la condición de los motores de combustión interna diésel, En ella se observa que del conjunto de herramientas del mantenimiento predictivo el $100 \%$ de los centros investigados cuenta con el servicio de escaneo de parámetros de motor, un $79 \%$ cuenta con el servicio de sonometría, un $66 \%$ cuenta con servicio de análisis de aceite, se notó la poca 0 nula aplicación de técnicas como vibraciones, gases y termografía. 
Figura 6. Aplicación de técnicas de diagnóstico del mantenimiento predictivo.

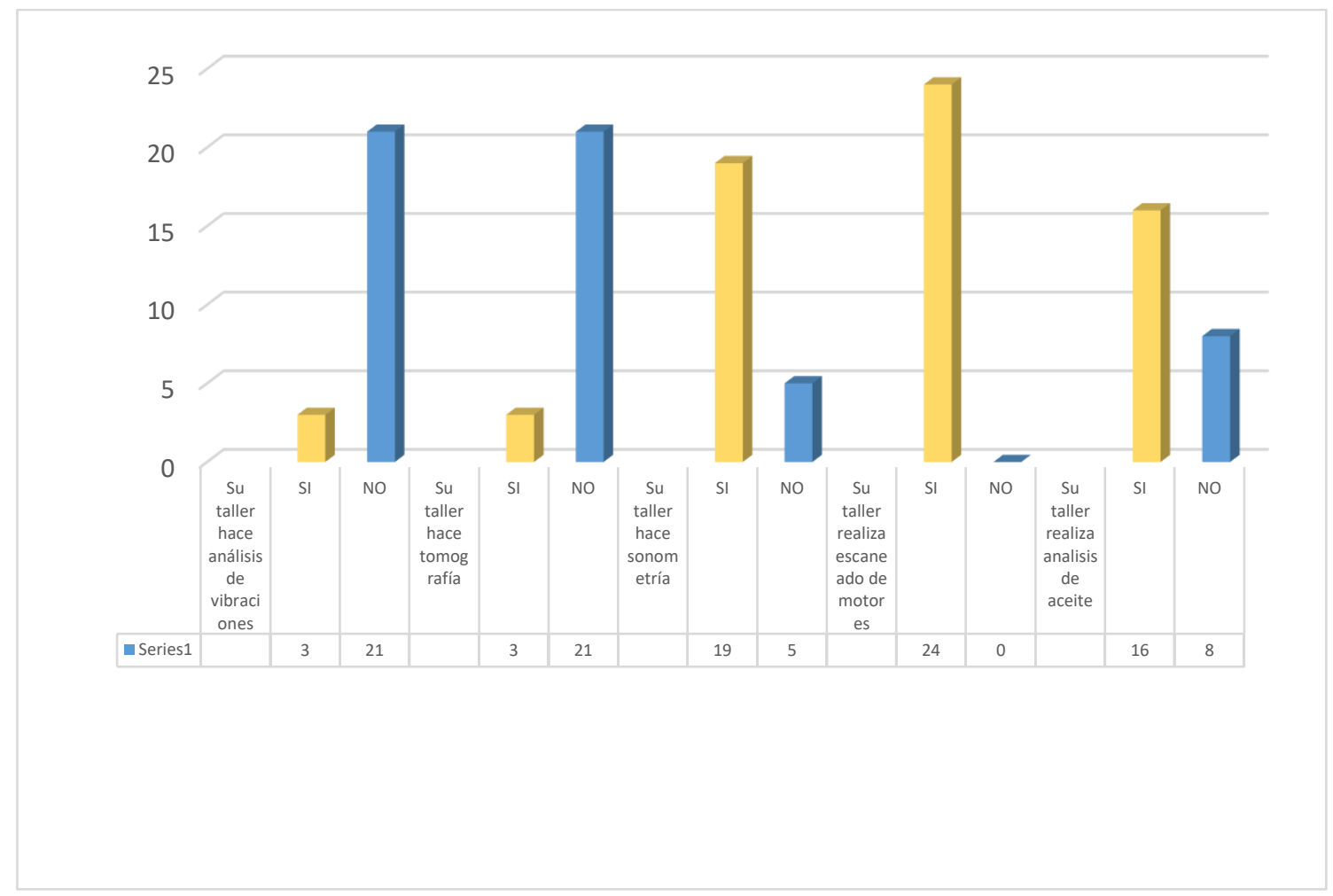

Fuente: Elaboración propia.

La entrevista permitió conocer el criterio empleado para realizar el mantenimiento predictivo, todos coinciden que la aplicación de la mayoría de las técnicas del mantenimiento predictivo principalmente es para la detección de fallas raras o no comunes y se aplican herramientas especiales como osciloscopio, compresimetro (vacuómetro), análisis de aceite, multímetro, entre otros y finalmente la gran mayoría coincide que la baja o nula aplicación de estas se debe principalmente a su elevado costo y baja demanda.
En la figura 7 se muestra grafico de distribución de los equipos disponibles según las diferentes técnicas encuestadas. En ella se observa que el $100 \%$ de los locales cuentan con un scanner de diagnóstico automotriz, un $56 \%$ posee equipo de diagnóstico por sonometría y un $46 \%$ de los talleres se encuentra equipado con otros instrumentos de mantenimiento entre los cuales están el laboratorio de inyectores, medidor de presión, multímetro automotriz entre los más destacados. 
Figura 7. Equipamiento tecnológico del taller.

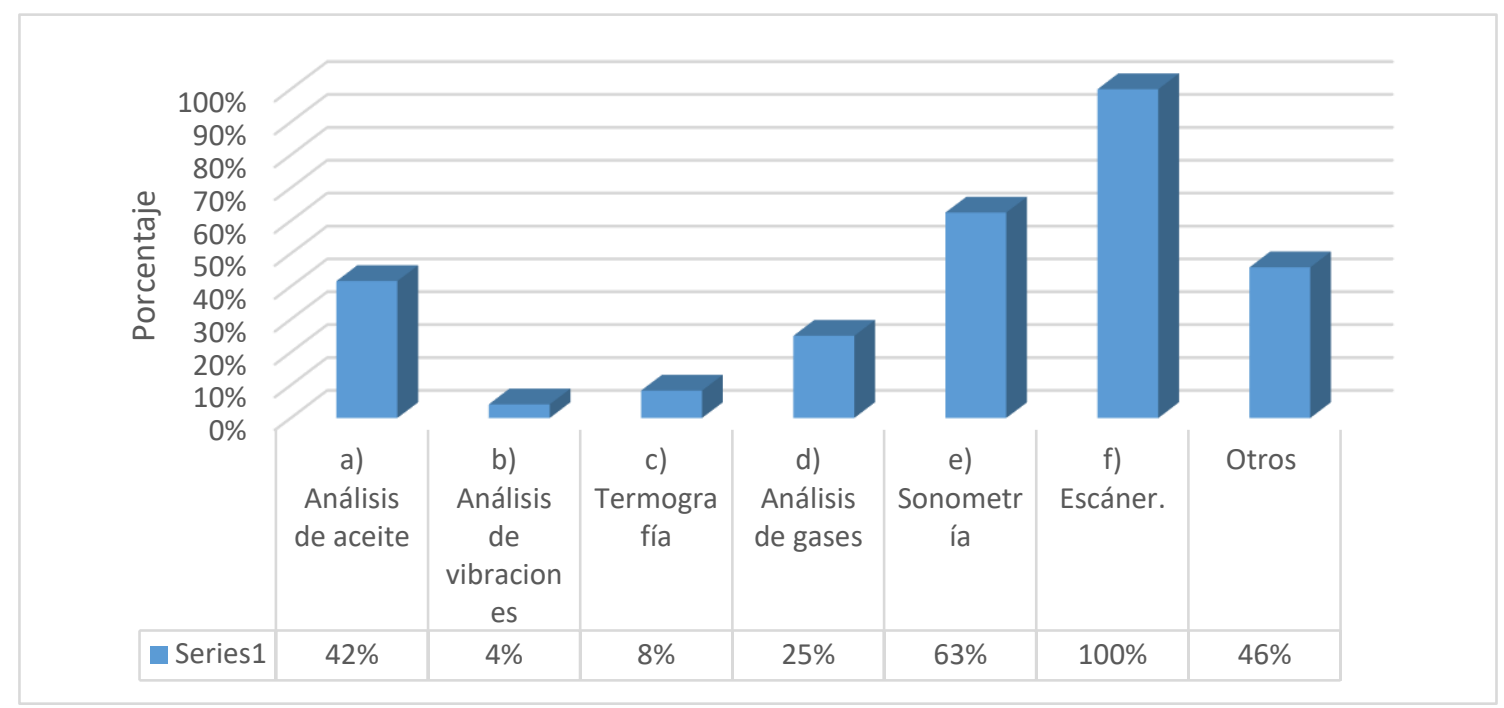

Fuente: Elaboración propia.

En la figura 8 se muestra el gráfico de la frecuencia de uso de las técnicas de diagnóstico y sus equipos. Como se puede observar la técnica con mayor frecuencia de uso es con $33 \%$ es el escáner automotriz y otros equipos como multímetro y compresimetro, y un $29 \%$ de talleres aplica con mayor regularidad el escáner automotriz y el estetoscopio automotriz.

Figura 8. Frecuencia de aplicación de equipos y técnicas de diagnóstico.

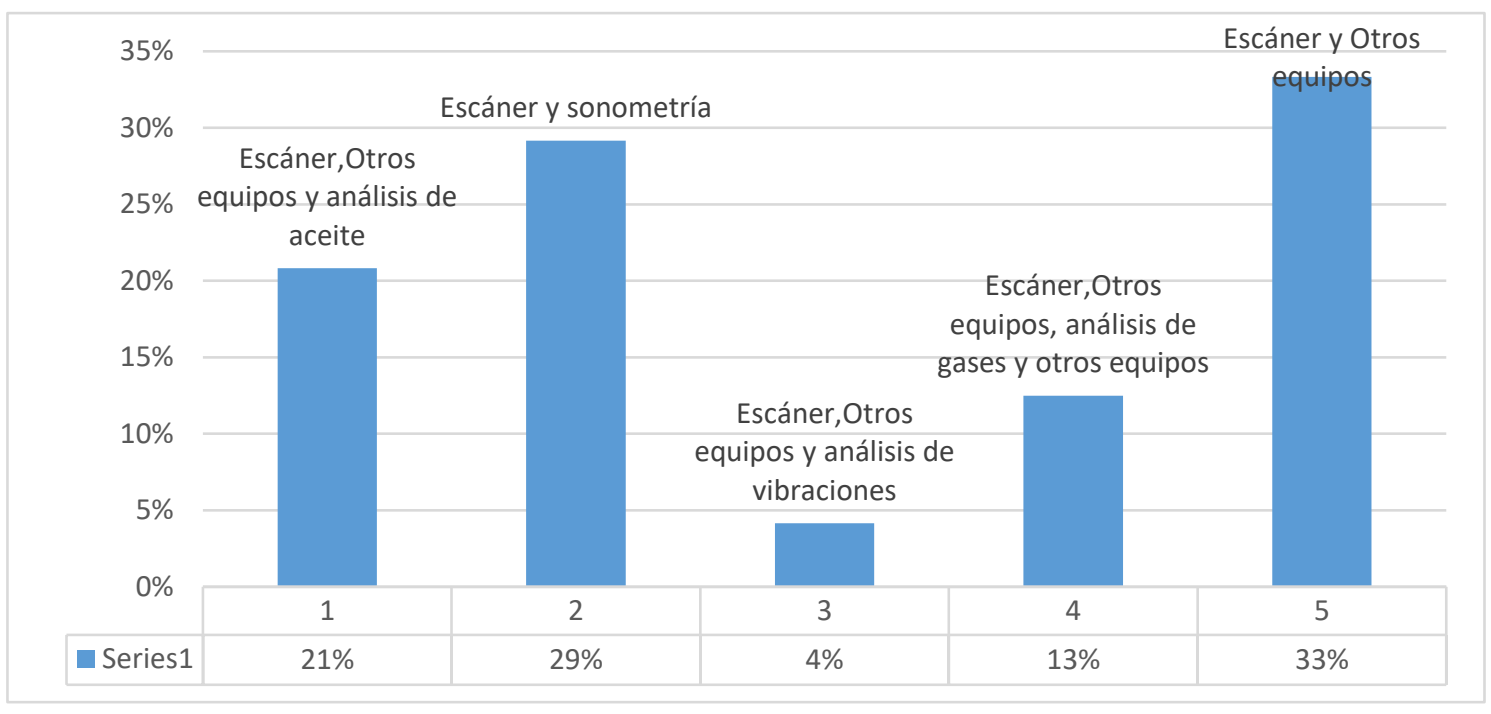

Fuente: Elaboración propia. 
La figura 9 muestra la distribución de los servicios ofertados por los talleres entrevistados, la aplicación de la entrevista arrojó que el $100 \%$ de los centros cuenta con equipo de diagnóstico para motores diésel, las ordenes de trabajo se encuentran principalmente compuestas por actividades de: Mantenimiento técnico programado, diagnóstico y reparación de avería de motor y ejecución de acciones preventivas; que un $88 \%$ del flujo vehicular se encuentra entre 0 a 5 unidades diarias y los fallos más comunes con los que acuden los clientes es en un $90 \%$ problemas con el sistema de alimentación, un 58 \% acuden por problemas eléctricos - electrónicos y 33 \% solicitan reparación de motor.

Figura 9. Servicios de mantenimiento ofertados.

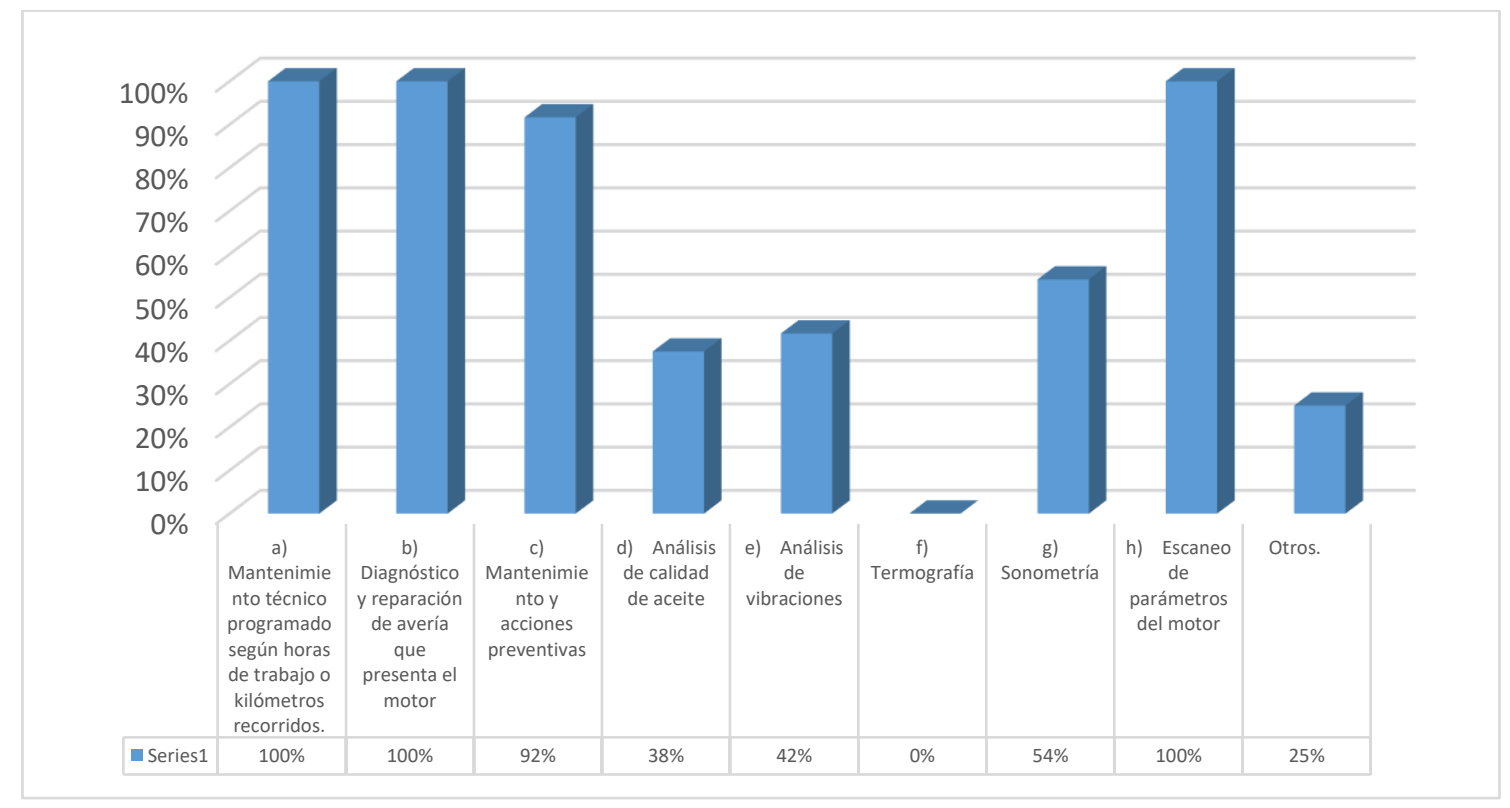

Fuente. Elaboración propia.

Como resultado de la aplicación de la entrevista a los talleres investigados de atención automotriz, proyecta que el equipamiento de diagnóstico que cuentan los talleres para la valoración de la condición y determinar las acciones de mantenimiento son los siguientes: Vacuómetro automotriz, estetoscopio

automotriz, osciloscopio automotriz, comprobador de hermeticidad, laboratorio de Inyección, medidor de turbo y MDI escáner de marca, entre los más destacados. 


\section{Conclusiones}

- Respecto al factor humano los resultados de la entrevista se puede dividir en dos grupos, por un lado los centros de atención al cliente de marcas reconocidas donde se encuentra que el personal de servicio cuenta con un nivel de preparación medio, acreditado por cursos y entidades de formación profesional y los directivos son profesionales, por otra parte el personal que presta servicio en los centros de atención 0 talleres mecánicos no asociados a marcas, se encuentra con un alto nivel de personal empírico en áreas de servicio, el personal administrativo es profesional y su participación en capacitaciones en muy poca.

- La entrevista arrojo que los equipos tecnológicos más relevantes que se encuentra con mayor frecuencia en los talleres de atención automotriz de la provincia son, por su orden, el escáner automotriz con un $100 \%$, multímetro automotriz con una presencia del $60 \%$ y el osciloscopio con un $30 \%$, siendo estas herramientas fundamentales para el diagnóstico, lo que indica un bajo nivel de tecnificación. por otro lado, a través de la observación directa se determinó que el $80 \%$ de talleres cuentan con elementos como elevadores, tecles entre otros; mientras en los centros de posventa existe un nivel alto de los instrumentos mencionados y la presencia de herramientas/técnicas especiales se encuentra de la siguiente manera: estetoscopio automotriz con un $63 \%$ y análisis de aceite con un $42 \%$, sin embargo, técnicas especiales como vibraciones, análisis de aceite, análisis de gases y termografía no se disponen en los talleres de atención automotriz.

- Los encuestados, esencialmente de talleres no asociados utilizan fundamentalmente el método empírico, para la determinación de fallas; en menor grado acuden a herramientas de apoyo como manuales técnicos, bolines de mantenimiento, entre otros, y no le dan el peso que se merecen a las técnicas del mantenimiento predictivo para determinar la condición técnica.

- Finalmente, de lo antes expuesto se puede concluir que el estado de la aplicación de las técnicas del mantenimiento 
predictivo en motores de combustión interna diésel, de los centros de servicio automotriz en Manabí es poco satisfactorio.

\section{Bibliografía}

AEDE. (2020). Sector automotor en cifras. Boletín No. 50. Quito: Asociación de empresas automotrices del Ecuador. Retrieved from https://www.aeade.net/wpcontent/uploads/2020/12/Bole tin-Sector-en-cifras-50resumen-en-espanol.pdf

Agencia europea del medio ambiente. (2016). Retrieved from Contaminación ambiental por gases derivados del petróleo.

Álava, G. (2020, noviembre 12). Preditec. Retrieved from Análisis de vibraciones: http://www.preditec.com/mant enimiento-predictivo/analisisde-

vibraciones/\#: :text=El\%20an $\%$ C3\%A1lisis\%20de\%20vibra ciones $\% 20$ es,fallos\%20mec $\%$ C3\%A1 nicos\%20en\%20m $\% \mathrm{C} 3 \% \mathrm{~A} 1$ quinas\%20rotativas.

ANUARIO-2020-AEADE-1.pdf. (s. f.). Recuperado 27 de septiembre de 2021, de https://www.aeade.net/wpcontent/uploads/2021/06/AN UARIO-2020-AEADE-1.pdf

Autodoc.es. (2020, noviembre 14). autodoc.es. Retrieved from
¿Qué es importante saber sobre los motores diésel?: https://www.autodoc.es/info/q ue-es-importante-sabersobre-los-motores-diesel

Ballesteros, F. (2017). La estrategia predictiva en el mantenimiento industrial. Preditécnico, 36-44. Retrieved from http://www.preditec.com/mant enimiento-predictivo/

Barrios, J. L., \& Calderón, S. M. (2018). Análisis y diagnóstico de los tipos de mantenimiento en la pequeña y gran minería aurífera en la subregión del Bajo Cauca, Antioquia. Revista Metalnnova, 1, 17-24.

Becerra, D., \& Molina, J. (2014). Caracterización de fallos en la ignición de un motor de combustión interna alterantivo diésel mediante el uso de la termografía infrarroja. Cuenca: Universidad Politécnica Salesiana, Sede Cuenca. Retrieved from https://dspace.ups.edu.ec/bits tream/123456789/6537/1/UP S-CT003164.pdf

Bennett, F. V. Y. (2017). ANÁLISIS TERMOGRÁFICO DE LA SUBESTACIÓN SAN LORENZO $Y$ SU ALIMENTADOR PRIMARIO PRINCIPAL PARA ELABORAR UN PLAN DE MANTENIMIENTO PREDICTIVO. 20. 
Buchelli, L., \& García, V. (2015). Detección temprana de fallas en motores de combustión interna a diesel mediante la técnica de análisis de aceite. Revista Ciencia UNEMI, 8(15), 84-95. Retrieved from http://repositorio.unemi.edu.e c/bitstream/123456789/3102/ 1/DETECCI\%c3\%93N\%20TE MPRANA\%20DE\%20FALLA S\%20EN\%20MOTORES\%20 DE\%20COMBUSTI\%c3\%93 N\%20INTERNA\%20A\%20DI ESEL\%20MEDIANTE\%20LA \%20T\%c3\%89CNICA\%20DE $\% 20$ AN\%c3\%81LISIS\%20DE \%20ACEITE.pdf

Calloni, J. C. (2004). Mantenimiento Electrico $\mathrm{Y}$ Mecanico Para Pequenas $Y$ Medianas Empresas/ Electrical and Mechanical Maintenance for Small and Medium Companies: Incluye Higiene $Y$ Seguridad Industrial. Nobuko.

Cárdenas, P., \& Cevallos, A. y. (2017). ANÁLISIS DE VIBRACIONES EN MOTORES DE COMBUSTIÓN INTERNA por medio de ultrasonido. Quito: Universidad Internacional del Ecuador. Retrieved from https://repositorio.uide.edu.ec /bitstream/37000/2187/1/TUIDE-1596.pdf

Cargua, S., \& Ipiales, M. (2014). Implemenatción de un plan de mantenimiento predictivo mediante la técnica de ultrasonido. Riobamba:
Escuela Superior Politécnica del Chimborazo. Retrieved from

http://dspace.espoch.edu.ec/ bitstream/123456789/4192/1/ 25T00246.pdf

Datos Geográficos. (s. f.). Gobierno de Manabí Ecuador. Recuperado 24 de septiembre de 2021, de https://www.manabi.gob.ec/sit io2020/datos-manabi/datosgeograficos

Delgado, J., Fygueroa, S., \& Bolívar, R. (2018). MONITORIZADO DE LA CONDICIÓN DEL ACEITE DE MOTORES DE automoción mediante un kit de pruebas rápidas. Revista colombiana de tecnologías de avanzada, 3(32,1), 42-49. Retrieved from http://revistas.unipamplona.e du.co/ojs_viceinves/index.php /RCTA/article/view/3303/1807

Errasti, M., Piloto, R., Ferrer, N., Melo, E., Werner de Dios, A., \& Goyos, L. (2013). Caracterización de un motor diesel trabajando con mezclas de aceite de Jatropha y combustible diesel. Energética, 34(3), 198-207. Retrieved from http://scielo.sld.cu/pdf/rie/v34 n3/rie03313.pdf

Estadísticas de ventas |
www.oica.net.
https://www.oica.net/category
/sales-statistics/


Evans, M. (2018, noviembre 24). Engine that powers the world. El motor que mueve al mundo. Londres, Inglaterra, Inglaterra: BBC Mundo. Retrieved from https://www.bbc.com/mundo/ noticias/2015/11/151124_eco nomia_diesel_como_cambio_ el_mundo_ms

Fernández, F. J. G. (2005). Teoría y práctica del mantenimiento industrial avanzado. FC Editorial.

García, S. (2017). ¿Es el mantenimiento predictivo la mejor o única alternativa para al plantear un plan de mantenimiento? IRIM Instituto RENOVETEC de Ingeniería del Mantenimiento, Ed. especial 6(1), 22-29. Retrieved from ¿Es el mantenimiento predictivo la mejor o única alternativa al plantear un plan de mantenimiento?:

http://renovetec.com/irim/revi sta/REVISTA_IRIM_NUMER O6_v1.pdf

García, S. (2019, junio 2). mantenimiento.renovetec.co m. Retrieved from Las diferentes técnicas de diagnóstico: http://www.mantenimiento.ren ovetec.com/185-\%20lasdiferentes-tecnicas-dediagnostico

Garrido, S. G. (2011). La contratación mantenimiento industrial: Procesos de externalización, contratos y empresas de mantenimiento. Ediciones Díaz de Santos.

Gaspar, K., Jordana, F., Martos, B., \& Puig, A. (2011). Estudi sobre la termografia infraroja aplicada a l'edificació. Recerca de possibilitat d'aplicació en façanes. Barcelona: Escola Politecnica Superior de Barcelona, UPC. Retrieved from https://upcommons.upc.edu/h andle/2099.1/12172?show=fu II

Gaviria, J., Cadena, D., Zuluaga, M., Rueda, C., González, C., Vélez, S., . . . Botero, M. (2018). ¿Hacia dónde tiende el mantenimiento? Estudio de un caso en la industria automotriz. EAFIT Colombia, 1-6. Retrieved from https://educacion.aciem.org/C IMGA/2018/Trabajos/2018025\%20TRA_COL_G_VILLE GAS_CIMGA2018.pdf

Gonzáles, D. (2006). Contribuciones A Las Técnicas No Destructivas para evaluación y prueba de procesos y materiales basadas en radiaciones infrarrojas. Santander: Universidad de Cantabris. Retrieved from https://www.tesisenred.net/bit stream/handle/10803/10706/ TesisDAGF.pdf?sequence $=1$ \&isAllowed $=\mathrm{y}$ 
González, P. D. (2013). Transporte y Logística Internacional. 73.

Guerrero, C. (2013). Evaluación de la Aplicabilidad de la Técnica de la Termografía Infrarroja al Reconocimiento del Estado de Elementos de Hormigón. Barcelona: Universitat Politécnica de Catalunya. Retrieved from https://upcommons.upc.edu/b itstream/handle/2099.1/19610 /CelyGuerrero_TFM.pdf

Gutiérrez, M. (2019). Medición de las vibraciones del motor utilizando mezclas de combustible de aceite vegetal reciclado y diesel. Un análisis matemático y computacional. INNOVA Research Journal, ISSN 2477-9024, 9-20. Retrieved from https://revistas.uide.edu.ec/in dex.php/innova/article/view/7 95/933

Gutiérrez, M., Íñiguez, J., Cadena, X., \& Santiana, G. (2017). Análisis De Las Vibraciones De Un Motor Ciclo Otto Con Una Mezcla Combustible A Base De Gasolina $Y$ De Etanol. INNOVA Research Journal, 2 (10.1), 138-146.

Hidalgo, E., \& Robles, F. (2016). Condiciones de monitoreo para sistema de control de motores de combustión interna. Ciencias Holguín, 22(3), 1-12.

Idrovo, j., \& Pugo, K. (2017). Sistema de toma de decisiones inteligentes para el mantenimiento preventivo $y$ predictivo del sistema de inyección de un motor de combustión interna del vehñiculo corsa evolution 1.4L. Cuenca: Universidad Politécnica Salesiana, Sede Cuenca. Retrieved from https://dspace.ups.edu.ec/ha ndle/123456789/14928

IMPLANTACIÓN DE UN SISTEMA DE MANTENIMIENTO PREDICTIVO BASADO EN CONDICION EN UNA PLATAFORMA NAVAL. (s. f.). Recuperado 7 de octubre de 2021, de https://repositorio.upct.es/bitst ream/handle/10317/6511/tfgmorimp.pdf?sequence=1\&isAllow $\mathrm{ed}=\mathrm{y}$

López, G., \& Basterra, A. (2010). Aplicación de la Termografía Infrarroja en la Evaluación no destrutiva de estructuras de madera. Valladolid: Universidad de Valladolid. Retrieved from https://www.worldcat.org/title/ aplicacion-de-la-termografiainfrarroja-en-la-evaluacionno-destructiva-deestructuras-demadera/oclc/688358761

Lucendo, J. (2019). Las Edades del Automóvil: Historia del Automóvil. Jorge Lucendo.

Macián, V., Tormos, B., Olmeda, P., \& Chacón, J. (2002). DIAGNÓSTICO DE 
MOTORES

DE

COMBUSTIÓN interna

alterantivos mediante oscilaciones de bloque. VIS Ingenierías, 1(2), 11-15. Retrieved from https://dialnet.unirioja.es/servl et/articulo?codigo $=6299725$

Maldague, X. (2001). Theory and Practice of Infrared Technology for Nondestructive Testing. Toronto: John Wiley \& Sons, INC.

MANTENIMIENTO PREDICTIVO: VENTAJAS DESVENTAJAS. (2019, enero 16). Valbor Soluciones. https://www.valborsoluciones. com/mantenimiento/manteni miento-predictivo-costoso/

Martínez, B. T. (2005). Diagnóstico de motores Diesel mediante el análisis del aceite usado. Reverte.

Martínez, D. V. (2017). MUESTREO PROBABILÍSTICO Y NO PROBABILÍSTICO. 14.

Motor.es. (2020, noviembre 12). motor.es. Retrieved from El motor diésel: funcionamiento y aplicaciones: https://www.motor.es/quees/motor-diesel

Ortiz, M. (2015). Estudio Comparativo del Uso del Diesel Entre Europa y Ecuador, utilizados para motores de vehículos. Quito: Universidad Internacional del
Ecuador. Retrieved from https://repositorio.uide.edu.ec /bitstream/37000/610/1/TUIDE-0560.pdf

Otero, A. (2019, noviembre 6). Xataca España. Retrieved from La realidad de la contaminación de los coches diésel:

https://www.xataka.com/auto $\mathrm{movil} /$ realidad-contaminacioncoches-diesel-que-errormeterlos-a-todos-saco

Rafael, M., \& Hernández, A. (2015). Caracterización de un motor de combustión interna con dos tipos de combustible. Sanfandila: Secretaría de comunicaciones transportes. Publicación Técnica No. 417.

Reina-Pérez, F. C., Reina-Quiñónez, F. M., Valencia-Ortiz, N. P., Chere-Quiñónez, B. F., \& Góngora-Ortiz, J. G. (2018). El mantenimiento predictivo, eficaz para sistemas eléctricos de potencia. Polo del Conocimiento, 2(12), 134.

Renovetec. (2020, noviembre 10). MantenimientoPetroquímica.c om. Retrieved from ¿QUÉ ES EL MANTENIMIENTO PREDICTIVO?:

http://www.mantenimientopetr oquimica.com/mantenimiento predictivo.html

Rodríguez, J. (1998). Diagnostico por vibraciones en motores de combustión interna. Ingeniería Mecánica 1(1), 11 - 
26. Retrieved from https://www.researchgate.net/ publication/296483914_Diagn ostico_por_vibraciones_en_m otores_de_combustion_intern a

Rosales, G. (2014). DISEÑO DE UN PROGRAMA INTEGRAL DE MANTENIMIENTO

MECÁNICO

PARA

MOTORES

HYUNDAI

9H21/32 BASADOS EN LAS NORMAS ISO 9001:2008, ISO 14001-2004 Y OHSAS 18001-2007. Guayaquil: Universidad de Guayaquil. Retrieved from http://repositorio.ug.edu.ec/bit stream/redug/7990/1/Tesis\% 20Rosales\%20T4AArial\%201.pdf

Siguenza, A., \& Paucar, A. (2016). Termografía aplicada al diagnóstico de un motor Hyunday diésel 2.0 CRDI de combustión interna alternativo como técnica de mantenimiento predictivo de fallos provocado por el sistema de alimentación aire combustible. Quito: Escuela Poliécnica Nacional.

Tormos, B. (2005). Diagnóstico de motores diésel mediante el análisis de aceite usado. Barcelona: Reverté. Retrieved from

https://books.google.es/books ?hl=es\&lr=\&id=DqJuqL_Uzjk $C \&$ oi=fnd $\& p g=P A 1 \& d q=T \% C$ $3 \% 89 \mathrm{CNICA}+\mathrm{DE}+\mathrm{ANALISIS}$ $+\mathrm{DE}+\mathrm{ACEITE}+\mathrm{EN}+\mathrm{MOTORE}$
S+DIESEL\&ots=kl7GhpkAr4 \&sig=OrjuYAZJREF16XnYxb W0T2plvJE\#v=onepage $\& q=T$ \%C3\%89CNICA\%20DE\%20 ANALISIS\%20DE\%20ACEIT E\%20EN\%20MOTORES\%20 DIESEL\&f $=f$

Velásquez, G., \& Rienstra, A. (2020, octubre $10)$. Reliabilityweb.com. Retrieved from Ultrasonido en activos móviles:

https://reliabilityweb.com/sp/a rticles/entry/ultrasonido-enactivos-moviles

Velásquez, J. A. M. (2018). Aplicación de RCM como estrategia de implementación del mantenimiento predictivo para la metodología TPM. 98.

Yanes, J. D., López, Á. H., \& de, J. (2019). Mantenimiento predictivo en el mundo marítimo. 2019, 66. 